\title{
Comparative study of hysteroscopy, hysterosalpingography and transvaginal sonography in evaluation of the female infertility
}

\author{
Shivangani Vohra*, Mamta Tyagi, Smriti Gupta, Esha Khanuja
}

\begin{abstract}
Department of Obstetrics and Gynecology, Subharti Medical College, Swami Vivekanand Subharti University,
\end{abstract} Meerut, Uttar Pradesh, India

Received: 09 December 2019

Accepted: 24 December 2019

*Correspondence:

Dr. Shivangani Vohra,

E-mail: vohrashivangani@gmail.com

Copyright: (c) the author(s), publisher and licensee Medip Academy. This is an open-access article distributed under the terms of the Creative Commons Attribution Non-Commercial License, which permits unrestricted non-commercial use, distribution, and reproduction in any medium, provided the original work is properly cited.

\section{ABSTRACT}

Background: One of the most important and underappreciated reproductive health problems in developing countries is that of infertility. Objective of this study was to evaluate HSC (hysteroscopy), HSG (hysterosalpingography) and ultrasonography in infertility.

Methods: This study was conducted among 100 infertile patients over a period of two years. The patients were questioned for the detailed history including socioeconomic status, medical history and previous history of taking any medications and supplements. The recruited patients had to undergo.

Results: Transvaginal sonography and clinical examination were done. Transvaginal sonography detected abnormality in $40 \%$ of women. 65 women had normal hysterosalpingography findings whereas the rest 35 women had abnormal hysterosalpingography findings. Thirty nine percent $(39 \%)$ had normal hysteroscopic findings while sixty one percent $(61 \%)$ had abnormal findings. The study depicts that hysteroscopy has sensitivity (95\%) and negative predictive value NPV $(92 \%)$ whereas that of TVS is $48.9 \%$ and $44.9 \%$ respectively. Hysterosalpingography has sensitivity $68.3 \%$ and NPV $40.3 \%$. Specificity (100\%) and positive predictive value PPV (100\%) of all the three modalities are the same.

Conclusions: The results of the present study showed that each modality provided useful information but no single modality provided complete information for evaluating infertile women. Thus, a combination of all three modalitiesTVS (transvaginal sonography), HSG (hysterosalpingography) and hysteroscopy is necessary to evaluate infertile women.

Keywords: Hysterosalpingography, Hysteroscopy, Infertility, Transvaginal sonography

\section{INTRODUCTION}

One of the most important and underappreciated reproductive health problems in developing countries is that of infertility. Infertility is a disease (an interruption, cessation, or disorder of body functions, systems, or organs) of the reproductive tract which prevents the conception of a child or the ability to carry a pregnancy to delivery (American Society for Reproductive Medicine (ASRM), 2012d). ${ }^{1}$ In India, eight to ten million couples are estimated to be childless. ${ }^{2,3}$
The causes of infertility in the female are ovulatory dysfunction $20 \%-40 \%$, tubal or peritoneal factor $20 \%$ $40 \%$, uterine factors $15 \%-20 \%$ and unexplained $20 \%$ $30 \% .^{4-6}$ None of the imaging modality alone is conclusive in diagnosing infertility. Transvaginal sonography, hysterosalpingography and hysteroscopy are all done for evaluation of infertility. ${ }^{7,8}$ No particular modality of investigation has been found to be better than the other. ${ }^{9}$ Hence the present study was taken up to evaluate the diagnostic accuracy of HSC (hysteroscopy) in 
comparison with HSG (hysterosalpingography) and ultrasonography in the evaluation of infertility.

\section{METHODS}

This study was conducted among 100 infertile patients attending gynecological OPD of Subharti Medical College, Meerut for infertility work-up, over a period of two years. The study protocol for all procedures was approved by the Institutional Review Board for Ethical Clearance of the institution and was performed in accordance with the Code of Ethics of the World Medical Association according to the Declaration of Helsinki of 1975, as revised in 2000. All patients were asked to sign a written consent form prior to commencement of the study. The subjects were selected according to the following inclusion and exclusion criteria:

\section{Inclusion criteria}

- Married women from reproductive age group of 1940 years with primary/secondary infertility, normal semen analysis of the husband, no evidence of haematological disorder/medical illness/surgical complications so as to avoid any anaesthetic or surgical risk during hysteroscopy were included in the study

\section{Exclusion criteria}

- Subjects having severe cardiac or respiratory illness, acute generalized peritonitis, allergy to iohexol dye, pelvic inflammatory diseases, women with ovarian or endocrinal cause of infertility were excluded from the study.

The patients were questioned for the detailed history including socioeconomic status, medical history and previous history of taking any medications and supplements. On TVS, the uterine anatomy and the adnexae were visualized using a $7.5 \mathrm{MHz}$ vaginal probe transducer. The contour of the endometrial stripe was assessed in the midline sagittal plane and the point of maximum thickness of the stripe (ET) was measured on a frozen image at $1.5 \times$ magnification. Appearance of the endometrial stripe was commented upon as either normal or abnormal; a specific note was made of any focal lesion seen in terms of impression of an endometrial polyp, submucous fibroid, intramural fibroid, or suspicion of hyperplasia.

HSG was performed after taking informed consent between day 7 to day 10 of last menstrual period. Appearance of uterus and tube was commented upon as either normal or abnormal; normal being an inverted triangle with well defined, smooth contours and spillage of dye from both the fimbrial ends. Specific note was made of any focal lesion seen in terms of impression of congenital abnormalities of uterine shape, luminal filling defects, abnormalities of uterine contour, blocked tubes, blocked cornu or beaded/dilated tubes. Hysteroscopy was done in endoscopic O.T. on an outpatient basis in the post menstrual phase. Systematic inspection of the cavity was performed and included examination of the fundus, anterior and posterior walls, lateral walls, both tubal ostia, and the lower uterine segment.

\section{Statistical analysis}

Data was collected in structured data collection forms. The data was collected in the proforma, tabulated in MS (Microsoft) excel chart and analyzed using SPSS (Statistical Package for the Social Sciences) software (version 22) using chi square test. Hysteroscopic findings were used as reference standards to calculate sensitivity and specificity in relation to hysterosalpingography as well as transvaginal sonography.

\section{RESULTS}

In our study majority of women (41\%) belonged to $26-30$ years age group. Out of 100 women, transvaginal sonography detected that sixty percent women had no pathology. Forty percent women had abnormal findings. 65 women had normal hysterosalpingography findings whereas the rest 35 women had abnormal hysterosalpingography findings. Thirty nine percent (39\%) had normal hysteroscopic findings while sixty one percent $(61 \%)$ had abnormal findings.

Table 1: Agreement of transvaginal sonography (TVS), HSG (hysterosalpingography) with hysteroscopy.

\begin{tabular}{|c|c|c|c|c|c|c|c|}
\hline \multirow{2}{*}{\multicolumn{2}{|c|}{ Variables }} & \multicolumn{2}{|c|}{ Hysteroscopy } & \multirow{3}{*}{$\begin{array}{l}\text { Total } \\
60\end{array}$} & \multirow{2}{*}{ Kappa value } & \multirow{2}{*}{ Chi square } & \multirow{2}{*}{ P-value } \\
\hline & & Normal & Abnormal & & & & \\
\hline \multirow{2}{*}{ TVS } & Normal & 22 & 38 & & \multirow{3}{*}{0.416} & \multirow{3}{*}{14.93} & \multirow{3}{*}{$<0.01 *$} \\
\hline & Abnormal & 17 & 23 & 40 & & & \\
\hline Total & & 39 & 61 & 100 & & & \\
\hline \multirow{2}{*}{ HSG } & Normal & 29 & 36 & 65 & \multirow{3}{*}{0.419} & \multirow{3}{*}{10.59} & \multirow{3}{*}{$<0.01 *$} \\
\hline & Abnormal & 10 & 25 & 35 & & & \\
\hline \multicolumn{2}{|l|}{ Total } & 39 & 61 & 100 & & & \\
\hline
\end{tabular}

*: statistically significant. 
In the comparative analysis between transvaginal sonography and hysteroscopy, the overall agreement between both the methods was moderate $($ kappa $=0.416)$ and significant correlation between the two with hysteroscopy being a better diagnostic tool (chi square value $=14.93$ and $\mathrm{P}$-value $<0.01$ ). Thus, the strength of agreement was considered to be moderate (Table 1).
The overall agreement between hysterosalpingography and hysteroscopy was found to be moderate (kappa = 0.419) and significant correlation was found with hysteroscopy being better diagnostic tool (chi square = 10.59, P-value <0.01). The strength of agreement is considered to be 'moderate' (Table 1).

Table 2: Diagnostic efficacy of TVS and hysterosalpingography in evaluating various pathologies in comparison to hysteroscopy.

\begin{tabular}{|c|c|c|c|c|c|c|}
\hline \multirow[b]{2}{*}{ Diagnostic tests (\%) } & \multicolumn{4}{|l|}{ TVS } & \multicolumn{2}{|l|}{ HSG } \\
\hline & Fibroids & Polyp & $\begin{array}{l}\text { Intrauterine } \\
\text { adhesions }\end{array}$ & $\begin{array}{l}\text { Uterine } \\
\text { anomaly }\end{array}$ & $\begin{array}{l}\text { Intracavitary } \\
\text { lesions }\end{array}$ & $\begin{array}{l}\text { Uterine } \\
\text { anomaly }\end{array}$ \\
\hline Sensitivity & 50 & 100 & 37.50 & 33.33 & 15.38 & 16.67 \\
\hline Specificity & 94 & 92.22 & 89.36 & 95.92 & 100 & 100 \\
\hline PPV & 50 & 58.82 & 37.50 & 33.33 & 100 & 100 \\
\hline NPV & 94 & 100 & 89.36 & 95.92 & 64.89 & 94.95 \\
\hline Accuracy & 89.29 & 85.47 & 81.82 & 92.31 & 62.60 & 65.10 \\
\hline
\end{tabular}

Table 3: Validity of hysteroscopy, hysterosalpingography and transvaginal sonography in infertility.

\begin{tabular}{|llllllll|} 
Group & $\begin{array}{l}\text { Sensitivity } \\
(\%)\end{array}$ & $\begin{array}{l}\text { Specificity } \\
(\boldsymbol{\%})\end{array}$ & $\begin{array}{l}\text { PPV } \\
(\boldsymbol{\%})\end{array}$ & $\begin{array}{l}\text { NPV } \\
(\%)\end{array}$ & $\begin{array}{l}\text { Positive likelihood } \\
\text { ratio }\end{array}$ & $\begin{array}{l}\text { Negative } \\
\text { likelihood ratio }\end{array}$ & Accuracy \\
\hline Hysteroscopy & 95 & 100 & 100 & 92 & 100 & 96 & 94 \\
\hline TVS & 48.9 & 100 & 100 & 44.9 & $0.91(0.65-1.27)$ & $1.16(0.71-1.87)$ & $45(35.03-55.27)$ \\
\hline HSG & 68.3 & 100 & 100 & 40.3 & $1.26(0.95$ to 1.67$)$ & $0.63(0.34$ to 1.16$)$ & $54(43.74$ to 64.02$)$ \\
\hline
\end{tabular}

TVS had sensitivity $50 \%$ while specificity of $94 \%$ in detecting fibroids. Transvaginal sonography in our study had sensitivity of $100 \%$, negative predictive value of $100 \%$, positive predictive value of $58.82 \%$ and specificity of $92.22 \%$ in detecting polyps. Specificity, sensitivity and accuracy in detecting uterine anomaly on sonography were $33.33 \%, 95.92 \%$ and 92.31 respectively (Table 2). Specificity and positive predictive value of hysterosalpingography in detecting intracavitary lesions are $100 \%$ for both HSG and hysteroscopy. Specificity, sensitivity, positive predictive value, negative predictive value and accuracy of hysterosalpingography in detecting uterine anomaly are $16.67 \%, 100 \%, 100 \%, 94.95 \%$ and 65.10 respectively (Table 2 ).

The study depicts that hysteroscopy has sensitivity (95\%) and negative predictive value NPV $(92 \%)$ whereas that of TVS is $48.9 \%$ and $44.9 \%$ respectively. Hysterosalpingography has sensitivity $68.3 \%$ and NPV $40.3 \%$. Specificity $(100 \%)$ and positive predictive value PPV $(100 \%)$ of all the three modalities are the same (Table 3).

\section{DISCUSSION}

In our study, hysterosalpingography revealed normal uterine cavity in sixty five percent $(65 \%)$ of the cases while thirty five percent $(35 \%)$ of the cases were abnormal. Similarly, Shukla P et al, in their study found that hysterosalpingography revealed normal uterine cavity in $65 \%$ of cases while $35 \%$ of cases abnormal in uterine cavity. ${ }^{5}$ In this study, the comparative analysis between transvaginal sonography and hysteroscopy, the overall agreement between both the methods was moderate. $($ Kappa $=0.416)$ and significant correlation between the two with hysteroscopy being a better diagnostic tool (chi square value $=14.93$ and P-value$<0.01)$. Thus, the strength of agreement was considered to be moderate. Similar results were reported by Shukla P et al. ${ }^{5}$ In this present study, the overall agreement between hysterosalpingography and hysteroscopy was found to be moderate (kappa $=0.419$ ) and significant correlation was found with hysteroscopy being better diagnostic tool. Shukla $\mathrm{P}$ et al, revealed moderate agreement between HSG and hysteroscopy with significant correlation was found with hysteroscopy being a better diagnostic tool. ${ }^{5}$ This study depicts that hysteroscopy has sensitivity (95\%) and NPV (92\%) whereas that of TVS is $48.9 \%$ and $44.9 \%$ respectively. Hysterosalpingography has sensitivity $68.3 \%$ and NPV $40.3 \%$. Specificity $(100 \%)$ and PPV $(100 \%)$ of all the three modalities are the same. These results were in accordance with the study done by Shukla $\mathrm{P}$ et al, who revealed that. ${ }^{5}$ The TVS was highly specific (100\%), but $51.21 \%$ sensitive compared with hysteroscopy 
(sensitivity $95.12 \%$, specificity $100 \%$ ). Similar to what was found by El- Mazny et al, in a study found transvaginal ultrasound had a low sensitivity of $41.7 \%$ and a high specificity. ${ }^{10}$

The diagnostic accuracy of HSG, the TVS and hysteroscopy to detect uterine abnormalities in infertile patient has been discussed by several authors. But it is still a matter of debate whether hysteroscopy should be performed routinely in infertile patients. There are many recommendations, some authors recommend routine diagnostic hysteroscopy, while others limit its use for infertile patients showing intracavitary alterations in the HSG and TVS. ESHRE guidelines indicate that hysteroscopy is unnecessary, unless it is used for confirmation and intrauterine treatment of suspected pathologies in previous studies. ${ }^{11}$

\section{CONCLUSION}

The results of the present study showed that hysteroscopy turned out to be the best modality in detecting pathologies in infertile women but lacked accuracy in detecting tubal pathologies, whereas HSG (hysterosalpingography) was the best modality for identifying tubal pathology. TVS (transvaginal sonography) was found to be most specific investigation in identifying any pathology. Each modality provided useful information but no single modality provided complete information for evaluating infertile women. Thus, a combination of all three modalities- TVS (transvaginal sonography), HSG (hysterosalpingography) and hysteroscopy is necessary to evaluate infertile women.

Funding: No funding sources

Conflict of interest: None declared

Ethical approval: The study was approved by the Institutional Ethics Committee

\section{REFERENCES}

1. American Society for Reproductive Medicine. Assisted reproductive technologies: a guide for patients, 2015. Available at: http://www.reproductivefacts.org/globalassets/rf/new s-and-publications/bookletsfact-sheets/english-factsheetsand-info-booklets/art.pdf. Accessed on $28^{\text {th }}$ March, 2019.

2. International Institute of population Sciences. National Family Health Survey; 1998-99, India. International Institute of population Sciences,
Mumbai, India. 2000. Available at: https://dhsprogram.com/pubs/pdf/FRIND2/FRIND2. pdf. Accessed on $22^{\text {th }}$ June 2019.

3. Talwar PP, Go OP, Murali IN. Prevalence of infertility in different population groups in India and its determinants. In: Statistics and Demography. New Delhi: National Institute of Health and Family Welfare and Indian Council of Medical Research; 1986. Available at: https://In:+Statistics+ and+demography\&author=PP+Talwar\&author $=\mathrm{OP}+$ Go\&author=IN+Murali\&publication_year=1986. Accessed on $28^{\text {th }}$ June 2019.

4. Dutta S, Guha R. A clinico-anatomical study on the etiological factors pertaining to primary infertility in females using some common investigative procedures. J Anat Soc India. 2007;56:14-7.

5. Shukla P, Yadav K, Mishra S. Hysteroscopic evaluation of uterine cavity in cases of infertility and its correlation with transvaginal ultrasound and hysterosalpingography. Int J Reprod Contracept Obstet Gynecol. 2016;5:3843-8.

6. Kamel RM. Management of the infertile couple: an evidence-based protocol. Reprod Biol Endocrinol. 2010;8:21.

7. Phillips $\mathrm{CH}$, Benson CB, Ginsburg ES, Frates MC. Comparison of uterine and tubal pathology identified by transvaginal sonography, hysterosalpingography, and hysteroscopy in female patients with infertility. Fertility Res Pract. 2015;1(1):20.

8. Niknejadi M, Haghighi H, Ahmadi F, Niknejad F, Chehrazi M, Vosough A, et al. Diagnostic accuracy of transvaginal sonography in the detection of uterine abnormalities in infertile women. Iranian J Radiol. 2012;9(3):139.

9. Benkaddour Y, Gervaise A, Fernandez H. Which is the method of choice for evaluating uterine cavity in infertility workup? J Gynecol Obstet Biol Reprod (Paris). 2010;39(8):606-13.

10. El-Mazny A, Abou-Salem N, El-Sherbiny W. Outpatient hysteroscopy: a routine investigation before assisted reproductive techniques? Fertil Steril. 2011;95(1):272-6.

11. Koskas M, Mergui J, Yazbeck C, Uzan S, Nizard J. Office hysteroscopy for infertility: a series of 557 cases consecutives. Obstet Gynecol Int. 2010;16:8096.

Cite this article as: Vohra S, Tyagi M, Gupta S, Khanuja E. Comparative study of hysteroscopy, hysterosalpingography and transvaginal sonography in evaluation of the female infertility. Int $\mathbf{J}$ Reprod Contracept Obstet Gynecol 2020;9:524-7. 